\title{
ANÁLISIS EPIDEMIOLÓGICO Y CLÍNICO DEL EMBARAZO ECTÓPICO: HOSPITAL BASE DE LOS ÁNGELES
}

\author{
Ignacio Cabrera Samith a , Iván Perales C. ${ }^{a}$, Germán Romero A. ${ }^{1}$ \\ 1 Servicio de Obstetricia y Ginecología, Hospital Base de Los Ángeles.

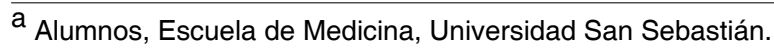

\section{RESUMEN}

Antecedentes: El embarazo ectópico (EE) se produce cuando el blastocisto se implanta en un sitio distinto al endometrio de la cavidad uterina, siendo el sitio más frecuente las trompas de Falopio. Objetivo: Conocer la incidencia y caracterizar el perfil de las pacientes con EE desde el punto de vista clínico, médico, quirúrgico y obstétrico. Método: Se realizó un estudio observacional analítico. La muestra fue obtenida a través de un muestreo no probabilístico consecutivo, obteniendo una muestra final de 70 pacientes. Resultados: El promedio de edad materna fue 30,6 años. El 78,6\% eran multíparas y $90 \%$ no usaban ningún método anticonceptivo. El $85,7 \%$ no tenían antecedentes de cirugías previas y el $91,4 \%$ no presentó antecedentes mórbidos. El 5,7\% tenía antecedentes de proceso inflamatorio pélvico, 18,5\% tabaquismo, $12,9 \%$ abortos previos, 14,3\% embarazo ectópico anterior. El manejo fue quirúrgico en 95,7\%. El 78,6\% presentó alguna complicación asociada, siendo la más frecuente el hematosalpinx. Conclusión: Los antecedentes epidemiológicos y clínicos de nuestra experiencia fueron concordantes con los registrados en la literatura y avalan nuestra conducta quirúrgica.

\section{PALABRAS CLAVE: Embarazo ectópico, salpingectomía, epidemiología}

\section{SUMMARY}

Background: Ectopic pregnancy (EP) occurs when the developing blastocyst becomes implanted at a site other than the endometrium of the uterine cavity. The most common extra-uterine location is the fallopian tube. Objective: The aim of our study is to know the incidence and the clinical, medical, obstetric and surgical profile of patients with EP. Method: An observational analytic study was made with a final sample of 70 patients. Results: Mean age was 30.6 years old, $78.6 \%$ were multiparas and $90 \%$ didn't use any contraceptive method. $85 \%$ had no history of previous surgery and $91.4 \%$ had no morbid background. $7.5 \%$ had history of pelvic inflammatory disease, $18.5 \%$ smoked, $12.9 \%$ had previous abortion and $14.3 \%$ previous EP. The management was surgical in $95 \%$ of patients; $78.6 \%$ presented an associated complication, being hematosalpinx the most often. Conclusion: The epidemiological and clinical background of our experience was consistent with those reported in the literature and guarantees our surgical treatment.

\section{KEY WORDS: Ectopic pregnancy, salpingectomy, epidemiology}




\section{INTRODUCCIÓN}

El embarazo ectópico (EE) se produce cuando el blastocisto se implanta en un sitio distinto al endometrio de la cavidad uterina. El sitio más frecuente de implantación es en las trompas de Falopio que ocurre en el $98 \%$ de todos los embarazos ectópicos (1). La prevalencia de EE entre las mujeres que acuden al servicio de emergencia con metrorragia durante el primer trimestre de embarazo, dolor o ambos es entre el 6 a 16\% (2). La incidencia global del EE ha aumentado desde mediados del siglo veinte, alcanzando a principio de los años noventa una cifra aproximada de 20 por 1000 embarazos (3), que ha sido asociado al aumento de la incidencia de la enfermedad inflamatoria pélvica (4).

Las manifestaciones clínicas clásicas ocurren generalmente entre 6 y 8 semanas después de la fecha de la última menstruación. Los síntomas clásicos del EE son el dolor abdominal (99\%), la amenorrea $(74 \%)$ y el sangrado vaginal $(56 \%)$. Estos síntomas pueden presentarse en EE rotos o no (5), siendo la taza de esta complicación de 18\% (6).

El diagnóstico de EE no complicado está basado en la sospecha clínica, apoyado en la ultrasonografía transvaginal (USTV) y la cuantificación de los niveles plasmáticos de la subunidad beta de la gonadotrofina coriónica humana (B-HCG). El diagnóstico mediante USTV se realiza al visualizar un saco gestacional extrauterino que contenga el saco vitelino o al embrión, sin embargo, estos hallazgos solamente se presentan en una proporción menor de EE (7). La observación mediante USTV de la presencia de masa anexial y útero vacío, en conjunto con una prueba de embarazo positiva, son los hallazgos más frecuentes en los EE no complicados. El rendimiento de la USTV para el diagnóstico de EE al visualizar útero vacío y masa anexial y/o un saco gestacional extrauterino, tiene una sensibilidad de $73,9 \%$ y una especificidad del $99 \%$ (8).

La B-HCG puede ser detectada en plasma u orina a partir del octavo día después del pico de LH si se ha producido el embarazo. El nivel plasmático de B-HCG, sobre el cual se debería observar el saco gestacional al interior de la cavidad uterina mediante ultrasonografía (US) es la llamada zona discriminatoria, cuyos valores fluctúan en la mayoría de los centros entre 1500-2000 UI/L para la USTV y sobre $6500 \mathrm{UI} / \mathrm{L}$ mediante US transabdominal (9). En un estudio representativo, el $98 \%$ de los embarazos intrauterinos fueron detectados mediante USTV en mujeres que presentaron valores de B-HCG sobre las $1500 \mathrm{UI} / \mathrm{L}(10)$.

El manejo actual del EE puede dividirse en médico, quirúrgico y expectante. El manejo médico consiste en la administración de diversos fármacos por vía sistémica o local destinado a la destrucción del tejido trofoblástico, el manejo quirúrgico que puede ser radical (salpingectomía) o conservador (salpingostomía) por laparotomía o laparoscopía, y la conducta expectante en espera de la evolución del cuadro (11).

El objetivo de nuestro estudio es conocer los aspectos epidemiológicos y clínicos de mujeres con EE tratadas en el Servicio de Ginecología y Obstetricia del Hospital Base Los Ángeles.

\section{PACIENTES Y MÉTODO}

Estudio observacional analítico de pacientes atendidas en el Servicio de Obstetricia y Ginecología del Hospital Base de Los Ángeles, con el diagnóstico de EE, ingresadas durante el período de enero a diciembre de 2008. La muestra fue obtenida a través de un muestreo no probabilístico consecutivo, formada por las mujeres que presentaron EE, diagnosticados mediante cuantificación de los niveles plasmáticos de B-HCG y USTV. Se obtuvo una muestra final de 70 pacientes. Las variables consignadas fueron: edad materna, métodos anticonceptivos, paridad, antecedentes mórbidos médicos y quirúrgicos, antecedentes de aborto previo, antecedente de embarazo ectópico anterior, antecedente de tabaquismo, niveles de B-HCG al momento del diagnóstico, edad gestacional al momento del diagnóstico, hallazgos ecográficos sugerentes de EE, tratamiento realizado (quirúrgico, médico o expectante), complicaciones asociadas, localización del EE.

Las variables fueron tabuladas en planilla electrónica Microsoft Excel 2007, y analizadas con el programa GraphPad Prism versión 5.00 de Windows. Para la comparación de variables categóricas se utilizó la prueba exacta de Fisher en las variables paridad y presencia de complicación. Para comparar medias se utilizó la prueba de T-Student con corrección de Welch en las variables: niveles de B-HCG, edad gestacional, edad materna en los grupos con y sin complicaciones. Se consideró significativo un valor $p<0,05$.

\section{RESULTADOS}

Antecedentes obstétricos generales: En 2008 se asistieron 3.125 partos y $70 \mathrm{EE}$. La incidencia de EE fue de 22,4/1000 partos. El promedio de edad fue de 30,6 $\pm 6,2$ años. El 21,4\% $(n=15)$ eran primigestas y $78,6 \%(n=55)$ multíparas. La paridad promedio fue de 1,2 $\pm 0,9$ gestaciones. El $90 \%(n=63)$ no usaban ningún método anticonceptivo (MAC), y 
$4,3 \%(n=3)$ usaban anticonceptivos orales (ACO) y $5,7 \%(n=4)$ usaban dispositivos intrauterinos (DIU). Antecedentes quirúrgicos: El $85,7 \%(n=60)$ no tenía antecedentes de cirugías previas, el 10\%(n=7) tenía antecedentes de salpingectomía anterior y el $1,4 \%(n=1)$ presentaba apendicectomía, colecistectomía o una cesárea.

Antecedentes mórbidos médicos: El 91,4\% ( $n=64)$ de la muestra no presentaba antecedentes mórbidos médicos. El 2,9\% $(n=2)$ tenían antecedentes de diabetes mellitus y el $1,4 \%(n=1)$ de hipertensión arterial.

Antecedentes mórbidos obstétricos: El 5,7\% ( $n=4)$ tenía antecedentes de proceso inflamatorio pélvico (PIP), 18,5\% (n=13) de tabaquismo, 12,9\% $(n=9)$ de abortos previos, y $14,3 \%(n=10)$ de embarazo ectópico anterior.

Antecedentes al diagnóstico: La edad gestacional promedio al momento del diagnóstico fue de 6 semanas con 5,9 días \pm 2 semanas con 3,5 días. Los niveles de B-HCG promedio fueron de 3.112,7 UI/L. Los hallazgos ecográficos sugerentes de embarazo ectópico fueron: líquido libre en fondo de saco de Douglas $10 \%(n=7)$, presencia de masa anexial $64,3 \%(n=45)$ y pseudosaco intrauterino $10 \%(n=7)$. El $98,6 \%(n=69)$ de los EE se localizaron a nivel tubario, el 1,4\% $(n=1)$ tuvo ubicación extratubaria a nivel del cuerno izquierdo (Figura 1).

Tratamiento: Fue quirúrgico en el 95,7\% ( $n=67)$, realizándose 36 salpingectomías derechas y 31 izquierdas, en el $4,3 \%(n=3)$ fue expectante.

Complicaciones: El 78,6\% $(n=55)$ de las gestaciones presentó alguna complicación asociada, siendo la más frecuente el hematosalpinx presente en el $57,1 \%(n=40)$ de los casos (Figura 2). No hubo diferencias significativas al comparar los valores de B-HCG entre los embarazos ectópicos con y sin complicaciones (Figura 3). La edad gestacional al momento del diagnóstico presentó diferencias significativas entre el grupo con complicaciones que presentó un promedio de 7,1 semanas $\pm 2,3$ y el

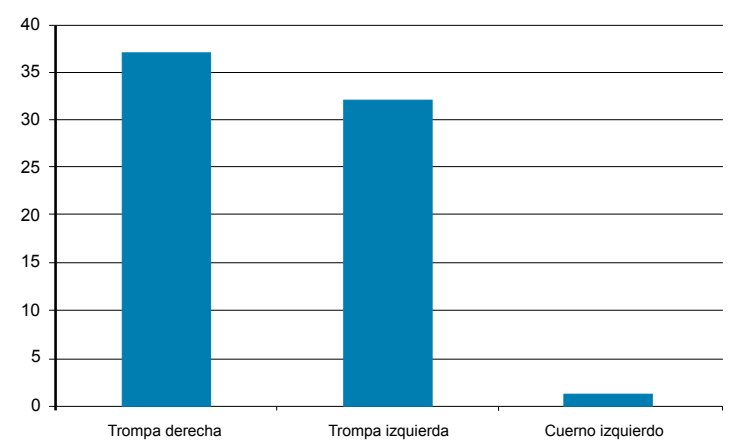

Figura 1. Localización de las gestaciones ectópicas. grupo sin complicaciones con un promedio de 6,3 semanas $\pm 2,3$ semanas. También hubo diferencia significativa en relación a la edad materna entre el grupo sin complicaciones con edad materna promedio de $30,7 \pm 4,9$ años y el grupo con complicaciones con edad materna promedio de 33,4 \pm 5,0 años. No hubo diferencias significativas en relación a la presencia de complicaciones asociadas entre las primigestas y multíparas.

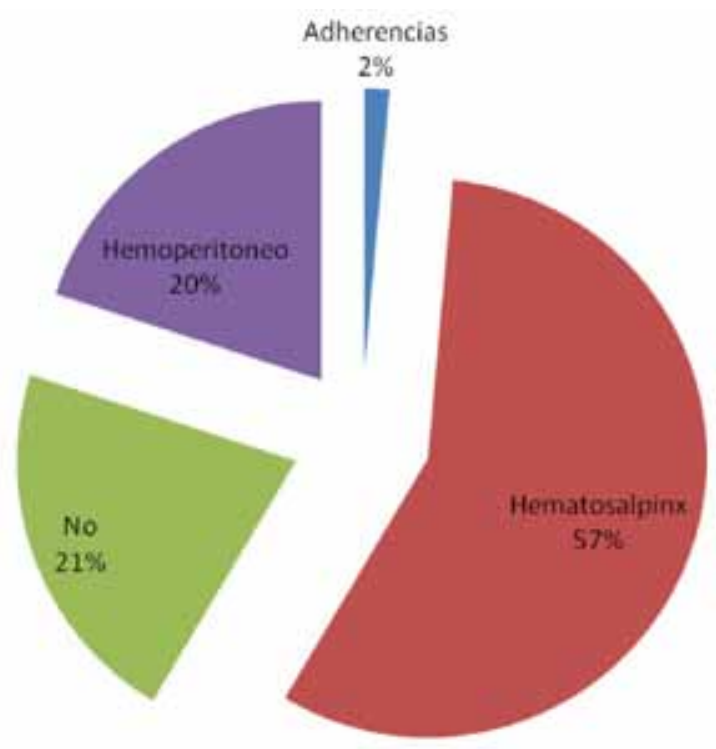

Figura 2. Tipo de complicaciones en los embarazos ectópicos.

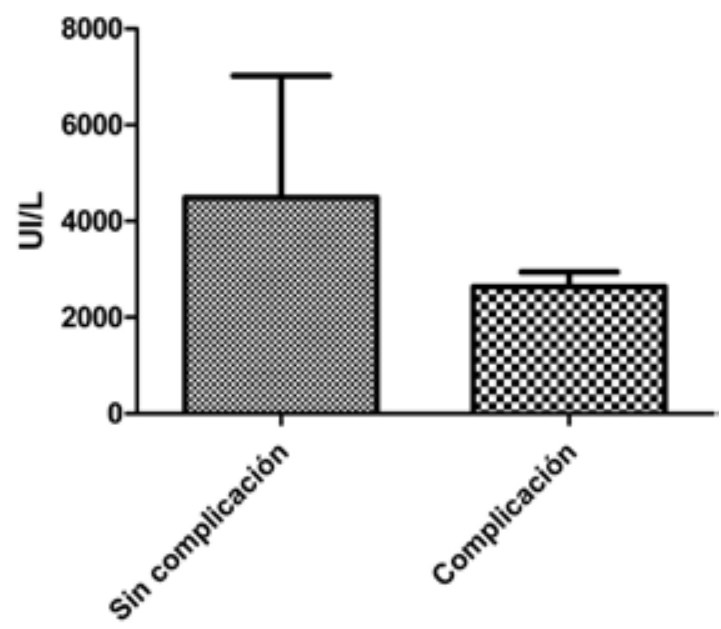

Figura 3. Niveles de B-HCG según presencia de complicaciones. 


\section{DISCUSIÓN}

La incidencia de EE reportada por las distintas series es variable, ya que muchas veces es difícil de precisar con exactitud en número total de gestaciones que se producen. En un estudio representativo realizado en Estados Unidos la incidencia de EE fue de 20/1000 embarazos (3), valor que se encuentra cercano al obtenido en nuestro estudio que tuvo una incidencia de 22,4 por 1000/partos.

Nuestro estudio muestra que las mujeres con diagnóstico de EE presentan un perfil clínico cuyo promedio etario fue de $30,6 \pm 6,2$ años, siendo la mayoría multíparas que no usaban ningún tipo de MAC. Los antecedentes quirúrgico de la muestra arrojó una baja prevalencia de antecedentes quirúrgicos, tan solo el 1,4\% tendría asociado el antecedente de salpingectomía previa, cirugía que ha sido asociado como un factor de riesgo conocido para EE, y que en estudios ha llegado a presentarse en un tercio de los casos (12). Dentro del perfil médico de la muestra, la mayoría no presentaba antecedentes mórbidos. Mientras que dentro de los antecedentes mórbidos obstétricos solamente el 5,7\% había presentado un PIP, evento que ha sido considerado como el principal factor de riesgo para EE, especialmente cuando la infección ha sido recurrente. En un estudio representativo se encontró un Odds Ratio para EE en mujeres que presentaron dos y tres o más episodios de PIP de 2,1 y 4,5 respectivamente (13).

El tabaquismo estuvo presente en el $18,5 \%$ de nuestra muestra, antecedente importante, ya que el consumo de cigarrillos en el periodo periconcepcional aumenta el riesgo de EE de manera dosis dependiente, también podría afectar a la inmunidad y a la motilidad de las trompas uterinas predisponiendo a las fumadoras a desarrollar un PIP (14). El antecedente de aborto previo se presentó en el $12,9 \%$ de nuestras pacientes, esto también es considerado un factor de riesgo para EE cuando va asociado a infertilidad, pues podría ser el reflejo de alteraciones anatómicas tubarias. El 14,3\% de nuestra muestra presentó el antecedente de EE anterior, cuyo riesgo se encuentra relacionado con la alteración tubaria que deja el EE inicial y el tipo de tratamiento que se eligió para su manejo (15).

El diagnóstico de EE en nuestro estudio se hizo entre la sexta y séptima semana de embarazo, lo que coincide con lo señalado por otros estudios, que señalan que el diagnóstico se hace entre la sexta y octava semana de embarazo (5). El diagnóstico de EE en nuestro estudio se basó en dos pilares, la USTV y la cuantificación plasmática de B-HCG. El examen mediante USTV mostró distintos hallazgos sugerentes de EE, siendo el más frecuente la presencia de una masa anexial con un $64,3 \%$, después vino la presencia de líquido libre en el fondo de saco de Douglas y la imagen de pseudosaco intrauterino, ambos con una frecuencia del $10 \%$.

Casi todas las mujeres con diagnóstico de EE deben ser manejadas con un tratamiento médico o quirúrgico, puesto que existe el riesgo potencial morbimortalidad materna por ruptura del EE. El tratamiento quirúrgico debe realizarse cuando existan signos de ruptura, niveles de B-HCG mayores a $5.000 \mathrm{UI} / \mathrm{L}$, necesidad de realizar laparoscopía para confirmar el diagnóstico y cuando se sospeche la presencia de un embarazo heterotópico (12). En nuestro estudio el $95,7 \%$ de los casos fueron manejados de manera quirúrgica; esto se debería a que la mayoría $(78,6 \%)$ de los EE presentó complicaciones asociadas.

La decisión de realizar una salpingostomía o una salpingectomía aún es controversial. Una revisión de estudios de cohorte, comparó la fertilidad resultante después de la realización de una salpingectomía o salpingostomía para manejo del $\mathrm{EE}$, no encontrándose diferencias entre el número de embarazos intrauterinos después de ambos procedimientos quirúrgicos, mientras que el riesgo de repetir un nuevo EE fue mayor al realizar una salpingostomía (16). La salpingectomía parece no afectar la tasa de embarazos intrauterinos en mujeres cuya trompa uterina contralateral se encuentra sana, y evita la complicación de EE recurrente en la misma trompa (17). En nuestro centro a todas las pacientes en que se optó por manejo quirúrgico se les realizó salpingectomía mediante laparotomía.

Otra opción es el manejo médico, que consiste en la administración de diversos fármacos, el más usado actualmente es el metotrexato, que es un antagonista del ácido fólico, que inhibe la síntesis de ADN y la reproducción de células con alta actividad proliferativa, como son las células neoplásicas, las trofoblásticas y las células embrionarias. Esta alternativa debe preferirse cuando no existe evidencia de ruptura, con niveles de B-HCG menores o iguales a $5000 \mathrm{UI} / \mathrm{L}$, con alza de la B-HCG en 48 horas, condiciones para seguimiento de corto y largo plazo y paciente con hemograma y función hepática normal (12).

La tercera opción de manejo es la conducta expectante, siempre que no exista evidencia de ruptura, niveles de B-HCG menores a $1500 \mathrm{UI} / \mathrm{L}, \mathrm{B}-\mathrm{HCG}$ descendente a las 48 horas y posibilidad de seguimiento inmediato (12).

En nuestro estudio no hubo diferencias significativas entre los niveles de B-HCG para la presencia de complicaciones. Sí hubo diferencias significativas de una edad gestacional mayor en el grupo 
que presentó complicaciones, esto estaría relacionado que con el aumento de los factores de riesgo con el paso de los años (18). Además, no se encontró diferencia para la presencia de complicaciones entre multíparas y primigestas.

\section{CONCLUSIÓN}

Los resultados clínicos y epidemiológicos de este estudio son concordantes con lo informado en la literatura, los que avalan nuestra conducta quirúrgica activa.

\section{BIBLIOGRAFÍA}

1. Tulandi T. Incidence, risk factors, and pathology of ectopic pregnancy. Update; mayo 2009. Disponible en: http://www.uptodate.com. Consultado el 4 de octubre de 2009.

2. Murray H, Baakdah H, Bardell T, Tulandi T. Diagnosis and treatment of ectopic pregnancy. CMAJ 2005;173:905-12.

3. Centers for Disease Control and Prevention (CDC). Ectopic pregnancy--United States, 1990-1992. MMWR Morb Mortal Wkly Rep 1995;44:46-8.

4. Kamwendo F, Forslin L, Bodin L, Danielsson D. Epidemiology of ectopic pregnancy during a 28 year period and the role of pelvic inflammatory disease. Sex Transm Infect 2000;76:28-32.

5. Alsuleiman SA, Grimes EM. Ectopic pregnancy: a review of 147 cases. J Reprod Med 1982;27:101-6.

6. Job-Spira N, Fernandez H, Bouyer J, Pouly JL, Germain E, Coste J. Ruptured tubal ectopic pregnancy: risk factors and reproductive outcome: results of a population-based study in France. Am J Obstet Gynecol 1999;180:938-44.

7. Condous G, Okaro E, Khalid A, Lu C, Van Huffel S, Timmerman $\mathrm{D}$, Bourne $\mathrm{T}$. The accuracy of transvaginal ultrasonography for the diagnosis of ectopic pregnancy prior to surgery. Hum Reprod 2005;20:1404-9.
8. Kirk E, Papageorghiou AT, Condous G, Tan L, Bora $S$, Bourne $T$. The diagnostic effectiveness of an initial transvaginal scan in detecting ectopic pregnancy. Hum Reprod 2007;22:2824-8.

9. Kadar N, DeVore G, Romero R. Discriminatory hCG zone: its use in the sonographic evaluation for ectopic pregnancy. Obstet Gynecol 1981; 58:156-61.

10. Barnhart KT, Simhan H, Kamelle SA. Diagnostic accuracy of ultrasound above and below the beta-hCG discriminatory zone. Obstet Gynecol 1999;94:583-7.

11. Álvarez M, Nannig C. Rol de la cirugía endoscópica en el manejo actual del embarazo ectópico tubárico. Rev Chi Obstet Ginecol 2005;70:15-20.

12. Farquhar CM. Ectopic pregnancy. Lancet2005;366:58391.

13. Hillis SD, Owens LM, Marchbanks PA, Amsterdam LF, Mac Kenzie WR. Recurrent chlamydial infections increase the risks of hospitalization for ectopic pregnancy and pelvic inflammatory disease. Am J Obstet Gynecol 1997;176:103-7.

14. Bouyer J, Coste J, Shojaei T, Pouly JL, Fernandez H, Gerbaud L, Job-Spira N. Risk factors for ectopic pregnancy: a comprehensive analysis based on a large case-control, population-based study in France. Am J Epidemiol 2003;157:185-94.

15. Yao M, Tulandi T. Current status of surgical and nonsurgical treatment of ectopic pregnancy. Fertil Steril 1997;67:421-33.

16. Hajenius PJ, Mol F, Mol BW, Bossuyt PM, Ankum WM, van der Veen F. Interventions for tubal ectopic pregnancy. Cochrane Database Syst Rev 2007;(1):CD000324.

17. Dubuisson JB, Morice P, Chapron C, De Gayffier A, Mouelhi T. Salpingectomy - the laparoscopic surgical choice for ectopic pregnancy. Hum Reprod 1996;11:1199-203.

18. Storeide O, Veholmen M, Eide M, Bergsjø P, Sandvei $R$. The incidence of ectopic pregnancy in Hordaland county, Norway 1976-1993. Acta Obstet Gynecol Scand 1997;76:345-9. 